\title{
Development of a GaAs Monolithic Surface Acoustic Wave Integrated Circuit
}

\author{
A. G. Baca, E. J. Heller, V. M. Hietala, S. C. Casalnuovo, G. C. Frye, J. F. Klem, and
}

T. J. Drummond

Sandia National Laboratories, P. O. Box 5800, MS-0603,

Albuquerque, NM 87185-0603, phone: (505)844-7127

\begin{abstract}
An oscillator technology using surface acoustic wave delay lines integrated with GaAs MESFET electronics has been developed for GaAs-based integrated microsensor applications. The oscillator consists of a two-port SAW delay line in a feedback loop with a four-stage GaAs MESFET amplifier. Oscillators with frequencies of 470, 350, and $200 \mathrm{MHz}$ have been designed and fabricated. These oscillators are also promising for other RF applications.
\end{abstract}

\section{INTRODUCTION}

Monolithic integration of chemical sensors with electronics will allow chemical detection instrumentation to be miniaturized, produced inexpensively, and have expanded functionality. The availability of such sensors will open up many new uses of chemical detectors because of greater portability and affordability. Chemical sensing through the use of acoustic wave devices has long been available using ST-quartz as the piezoelectric material for generating acoustic waves [1]. However, ST-quartz is not a suitable substrate for monolithic integration of microelectronics. GaAs is a piezoelectric material on which surface acoustic wave (SAW) devices can be fabricated and can also provide electronic capability. In this work, we demonstrate an oscillator technology using SAW delay lines integrated with GaAs MESFET electronics. This device is a major step toward the development of integrated chemical microsensors. Monolithic SAW oscillator technology has been proposed previously [2], but to our knowledge has not yet been demonstrated.

\section{PROPERTIES OF PIEZOELECTRIC MATERIALS}




\section{DISCLAIMER}

This report was prepared as an account of work sponsored by an agency of the United States Government. Neither the United States Government nor any agency thereof, nor any of their employees, make any warranty, express or implied, or assumes any legal liability or responsibility for the accuracy, completeness, or usefulness of any information, apparatus, product, or process disclosed, or represents that its use would not infringe privately owned rights. Reference herein to any specific commercial product, process, or service by trade name, trademark, manufacturer, or otherwise does not necessarily constitute or imply its endorsement, recommendation, or favoring by the United States Government or any agency thereof. The views and opinions of authors expressed herein do not necessarily state or reflect those of the United States Government or any agency thereof. 


\section{DISCLAIMER}

Portions of this document may be illegible in electronic image products. Images are produced from the best available original document. 
Piezoelectric materials can provide a convenient way to launch surface acoustic waves because of a coupling between strain and electric polarization fields. To launch an acoustic wave, an electric potential is used to create elastic deformations in a piezoelectric material by means of a voltage applied to an interdigitated transducer (IDT), which consists of a set of closely spaced metal electrodes deposited on the substrate. SAW devices have achieved commercial prominence for the better known piezoelectric materials ST-quartz and lithium niobate in such applications as oscillators for clocks and RF filters for TV tuners and wireless communications [3]. ST-quartz and lithium niobate are attractive materials for these applications because of some key material properties that are indicated in Table 1 . The acoustic velocity along with the desired frequency of operation determine the wavelength and hence the electrode separation within the IDT. GaAs, ST-quartz and lithium niobate all have similar acoustic velocities. The electromechanical coupling coefficient, $\mathrm{K}^{2}$, is smaller for GaAs than either ST-quartz or lithium niobate. Because a smaller $\mathrm{K}^{2}$ value will result in a weaker acoustic signal for a given input, the insertion loss for GaAs SAW devices will be greater than for ST-quartz or lithium niobate, requiring increased amplification of the acoustic signal and possibly smaller signal to noise ratio. Another important property is the temperature coefficient of delay (TCD), a measure of signal drift with temperature, which is optimum for ST-quartz at room temperature. The material properties of Table 1 may suggest that GaAs is not the favored piezoelectric material for conventional applications. However, GaAs is a good choice for integrating electronics with acoustic functions.

\section{SENSOR TECHNOLOGY}

A brief description of chemical sensing using SAW devices are described in this section, beginning with a description of a SAW delay line oscillator. An RF electrical signal applied to an input transducer is used to launch an acoustic wave. The wave propagates along the path between input and output transducers, termed a delay line, and is received at an output transducer. An amplifier in a feedback loop with the SAW delay line is used to construct an oscillator. The amplifier must be designed for sufficient gain at the desired oscillator frequency to overcome the SAW insertion loss. The acoustic velocity along with the desired 
oscillation frequency is used to calculate the spacings of the IDT electrodes. More details of the design of SAW delay lines are covered in reference 1.

Sorbent films (typically polymers) are deposited on the SAW delay line to enable chemical sensing. Acoustic coupling between the substrate surface and the polymer film changes the acoustic velocity in proportion to the mass of the sorbent film [1]. Chemical sensing comes about because molecules that absorb well on, or in, the sorbent film increase its mass and are detected as either a frequency shift of the oscillator or as a phase shift measured relative to an uncoated reference delay line.

Chemical sensors made with both ST-quartz and GaAs SAWs have been investigated with hybrid electronic assemblies [4]. The advantages of a monolithic GaAs-based approach are mainly related to size, performance, reliability, and cost. Reliability and cost are issues that typically motivate high integration levels. In chemical sensing applications, size is especially important because an integrated microsensor may consist of arrays of SAW delay lines each with separate polymer coatings to allow for sensitivity to different types of chemicals. The overall size reduction can be substantial for a large number of sensors. Some size reduction will occur from monolithic integration of acoustic and electronic functions. Even greater size reduction will result from the use of the acoustic devices scaled to higher frequencies, since the both the IDT and passive electronic elements (mainly capacitors and inductors) will be smaller. In addition, higher frequency leads to greater chemical sensitivity in SAWs [1]. At high frequencies, monolithic integration is also advantageous in terms of keeping RF signals on-chip as DC-in, DC-out is favorable for system integration.

\section{MESFET TECHNOLOGY}

This initial technology demonstration was made by modifying a conventional MESFET process with one additional processing step to deposit a metal film for IDT formation. The minimum IDT features of 1.5 $\mu \mathrm{m}$ result in a maximum oscillator frequency of $500 \mathrm{MHz}$. All GaAs-based electronics such as MESFETs, HEMTs, and HBTs are suitable at least up to several GHz. MESFETs were chosen because of their 
simplicity in fabrication and their wide commercial applicability. An epitaxial channel design was used in this work for convenience; ion implanted MESFETs are also suitable.

The MESFET structure consists of a $3000 \AA$ p-type confinement layer doped to $5 \times 10^{15} \mathrm{~cm}^{-3}$, a $800 \AA$ GaAs channel layer doped to $2.5 \times 10^{17} \mathrm{~cm}^{-3}$ and a $50 \AA$ undoped GaAs cap, all grown on a semiinsulating GaAs substrate by molecular beam epitaxy. A self-aligned refractory gate MESFET process was used for fabrication as illustrated in Fig. 1. In step 1, a W/WSi gate with a 0.7 mmgate length was deposited and patterned by reactive ion etching [5]. Shallow self-aligned Si source and drain implants then followed (step 2). The conditions used were ${ }^{29} \mathrm{Si}$ at $50 \mathrm{keV}$ and $1.1 \times 10^{13} \mathrm{~cm}^{-2}$. By keeping the energy to $50 \mathrm{keV}$, a more elaborate sidewall process can be avoided for this gate length. Ohmic contacts with $\mathrm{Ge} / \mathrm{Au} / \mathrm{Ni} / \mathrm{Au}$ were deposited and alloyed in step 3. Isolation of the active areas was accomplished by an oxygen implant (step 4). Next, the IDT metal was deposited. This metal layer was also used as for capacitor electrodes and as an interconnect metal. A $4300 \AA$ silicon nitride layer combined as an interlevel dielectric and a capacitor dielectric with reactive ion etching for vias and capacitor patterning. Processing was completed with the second interconnect metal patterning and deposition (steps 6 and 7). All lithography was done with conventional optical means. The source and drain ion implants were also used for resistor formation.

The electrical characteristics of a $0.7 \times 50 \mu \mathrm{m}^{2}$ enhancement-mode MESFET are shown in Fig. 2 and Fig 3. The device shows a DC output conductance of $5-7 \mathrm{mS} / \mathrm{mm}$ and a peak transconductance of 270 $\mathrm{mS} / \mathrm{mm}$ with a threshold voltage of $0.06 \mathrm{~V}$. The use of an epitaxial channel resulted in good uniformity of the threshold voltage.

\section{OSCILLATOR DESIGN AND OPERATION}

The GaAs-based oscillator consists of a four-stage MESFET amplifier combined in a feedback loop with a SAW delay line as shown in Fig. 4 for a $470 \mathrm{MHz}$ design. This oscillator is a simple demonstration of technology that can be used for chemical sensing capability and may also be promising for RF oscillator and filter applications. Passive elements included capacitors and resistors. No inductors were used because of 
their large size at this frequency. The amplifier was designed for $40 \mathrm{~dB}$ of gain to overcome the insertion loss of the SAW delay line, which is typically $30-35 \mathrm{~dB}$ at this frequency. On chip bypass capacitors (not shown in schematic) of $2 \mathrm{pF}$ and $4 \mathrm{pF}$ were included for the gate and drain supplies, respectively. A picture of a fabricated device is shown in Fig. 5. The oscillator size is approximately $1.9 \times 1.2 \mathrm{~mm}^{2}$.

The oscillator was tested on wafer with an HP spectrum analyzer with $V_{d}$ approximately $3 \mathrm{~V}$ and $\mathrm{V}_{\mathrm{g}}$ approximately $0.3 \mathrm{~V}$. The oscillator functioned at $471.86 \mathrm{MHz}$ with narrow line operation, as shown in Fig. 6. The power consumption was $50-60 \mathrm{~mW}$. The $200 \mathrm{MHz}$ and $350 \mathrm{MHz}$ oscillators with a similar design were also operational. The chip size for these designs are $3.5 \times 1.6 \mathrm{~mm}^{2}$, and $2.1 \times 1.3 \mathrm{~mm}^{2}$. The SAW delay line represents a larger fraction of the chip size for the lower frequencies. However, electronic functions are likely to take up more space in future designs.

\section{CONCLUSION}

Monolithically integrated SAW MESFET oscillator technology has been demonstrated. This technology will enable integrated microsensing applications and may be promising for RF oscillator and filter applications as well.

\section{ACKNOWLEDGMENTS}

The authors would like to thank Geraldine Lopez and Melissa Cavaliere for fabrication. Sandia is a multiprogram laboratory operated by Sandia Corporation, a Lockheed Martin Company, for the United States Department of Energy under Contract DE-AC04-94AL85000. 


\section{REFERENCES}

[1] D. S. Ballantine, R. M. White, S. J. Martin, A. J. Ricco, E. T. Zellers, G. C. Frye, H. Wohltjen, Acoustic Wave Sensors Theory Design, and Physico-Chemical Applications, Academic Press, San Diego, 1997.

[2] T. W. Grudkowski, G. K. Montress, M. Gilden, and J. F. Black, "Integrated Circuit Compatible Surface Acoustic Wave Devices on Gallium Arsenide," IEEE Trans. Microwave Theory and Techniques, vol. 29, pp. 1348-1356, 1981.

[3] C. K. Campbell, Surface Acoustic Wave Devices for Mobile and Wireless Communication, Academic Press, San Diego, 1998.

[4] G. Frye, R. Kottenstette, E. Heller, J. Brinker, S. Casalnuovo, A. Sellinger, N. Raman, and Y. Lu, "Optimizing Surface Acoustic Wave Sensors for Trace Chemical Detection," Proc. Transducers '97, vol. 2. p. 1322, IEEE, Piscataway, NJ, 1997.

[5] R. J. Shul, M. E. Sherwin, A. G. Baca, J. C. Zolper, and D. J. Rieger, "Short Gate Etching of W/WSi Bilayer Gates," Elect. Lett., vol. 32, pp. 70-71, (1996). 


\section{FIGURE CAPTIONS}

Fig. 1. A schematic representation of a self-aligned GaAs MESFET process for monolithic integration with acoustic wave devices.

Fig. 2. IDS-VDS for a self-aligned $0.7 \times 50 \mathrm{~mm}^{2} \mathrm{GaAs}$ MESFET.

Fig. 3. IDS $-V_{G}$ at $V_{D S}$ of $1.5 \mathrm{~V}$ for a self-aligned $0.7 \times 50 \mathrm{~mm}^{2} \mathrm{GaAs}$ MESFET.

Fig. 4. Schematic of a $470 \mathrm{MHz}$ SAW oscillator circuit.

Fig. 5. A microphotograph of a $470 \mathrm{MHz}$ GaAs SAW oscillator.

Fig. 6. The frequency spectrum of a GaAs SAW oscillator operating at $471.86 \mathrm{MHz}$. 


\section{TABLES}

Table 1. Several material properties of some common piezoelectric materials. K2 is the electromechanical coupling coefficient and TCD is the temperature coefficient of delay.

\begin{tabular}{|l|c|c|c|}
\hline $\begin{array}{l}\text { Piezoelectric } \\
\text { Material }\end{array}$ & $\begin{array}{c}\text { Acoustic } \\
\text { Velocity } \\
(\mathrm{m} / \mathrm{sec})\end{array}$ & $\begin{array}{c}\mathrm{K}^{2} \\
(\%)\end{array}$ & $\begin{array}{c}\mathrm{TCD} \\
(\mathrm{ppm} / \mathrm{C})\end{array}$ \\
\hline $\mathrm{LiNbO}_{3}$ & 3480 & 4.5 & 80 \\
\hline ST-quartz & 3160 & 0.11 & 0 \\
\hline GaAs(001) & 2860 & 0.07 & 50 \\
\hline
\end{tabular}


1. Gate Metal

冝

2. Source/Drain Implants and RTA

twterth

3. Ohmic Metal

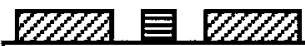

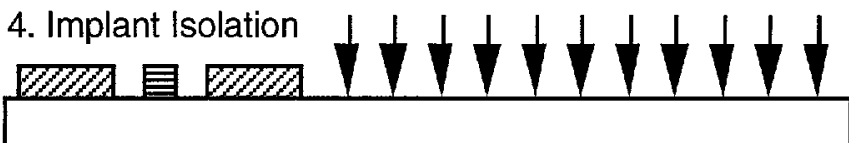

5. IDT and Lower Capacitor Metal

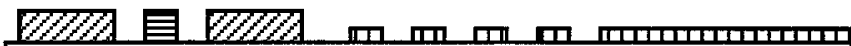

6-7. Dielectric and Interconnect Metal

W

Figure 1. 


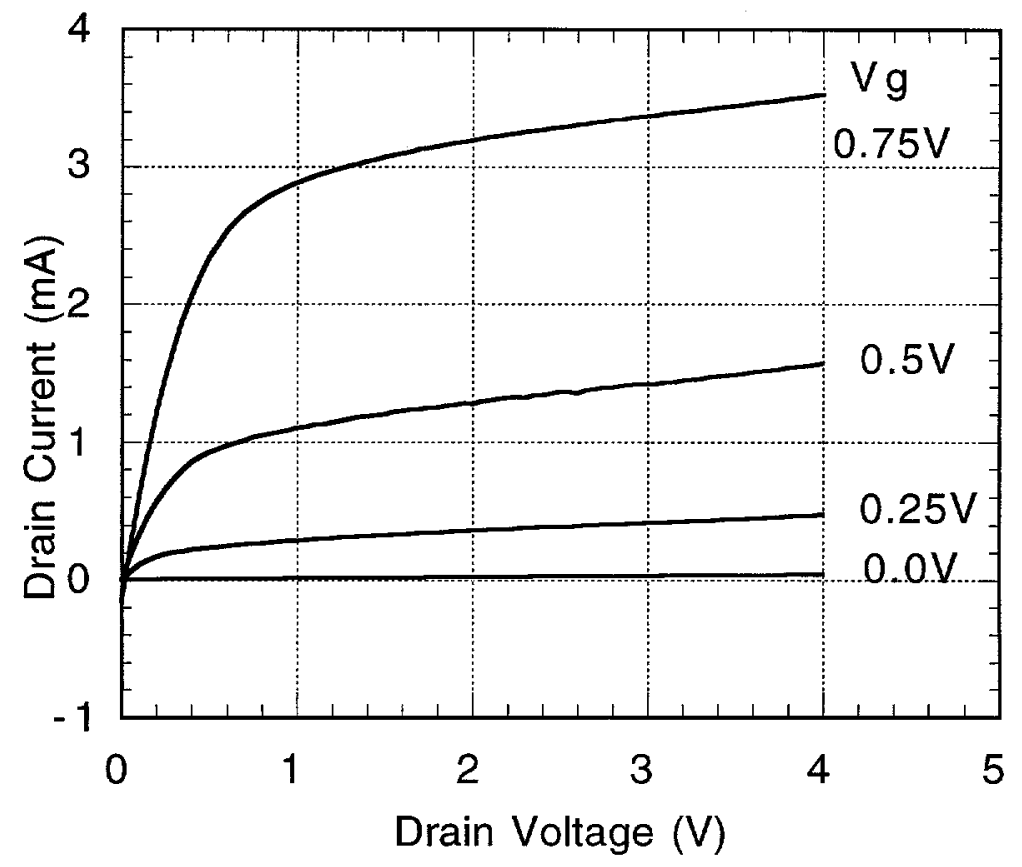

Figure 2. 


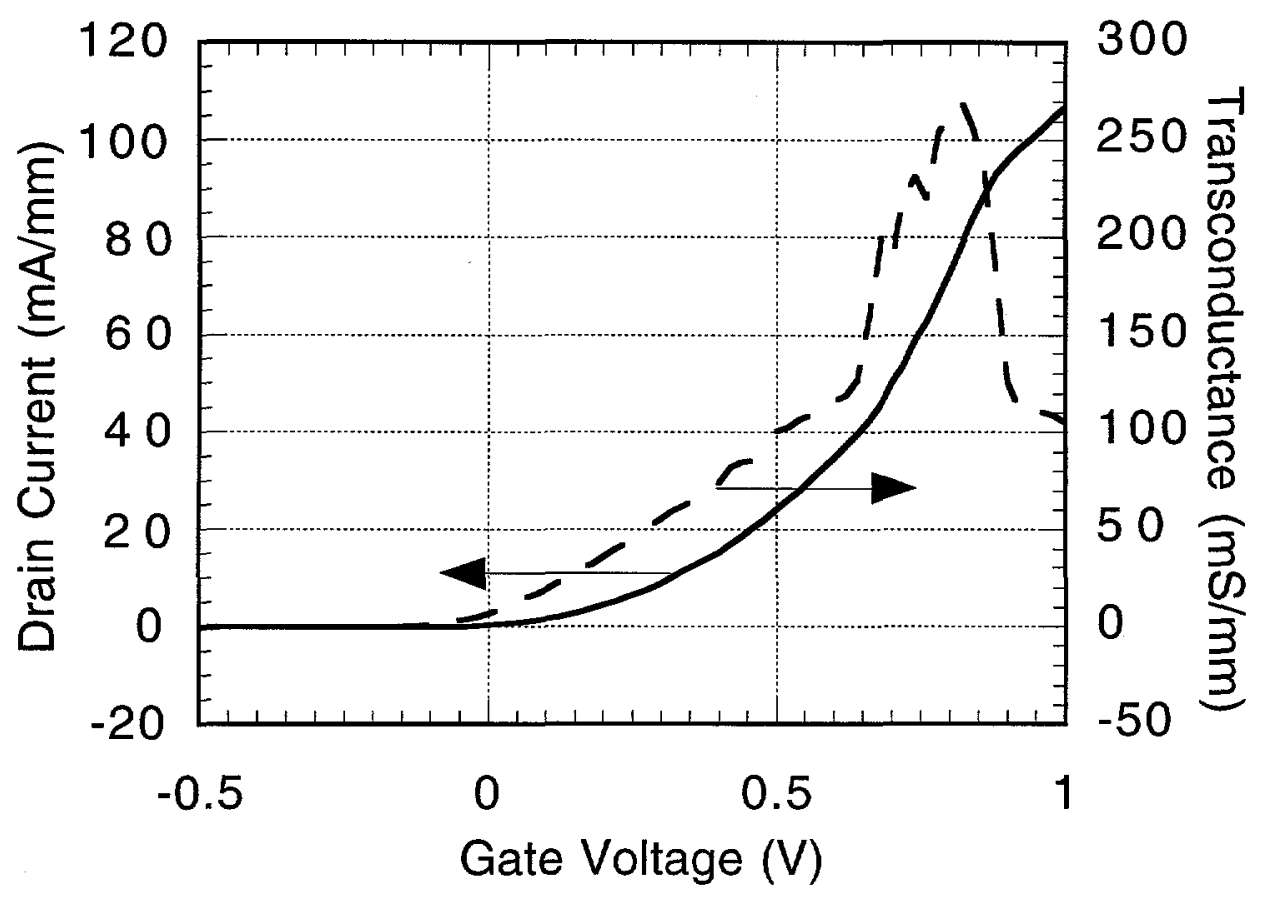

Figure 3. 


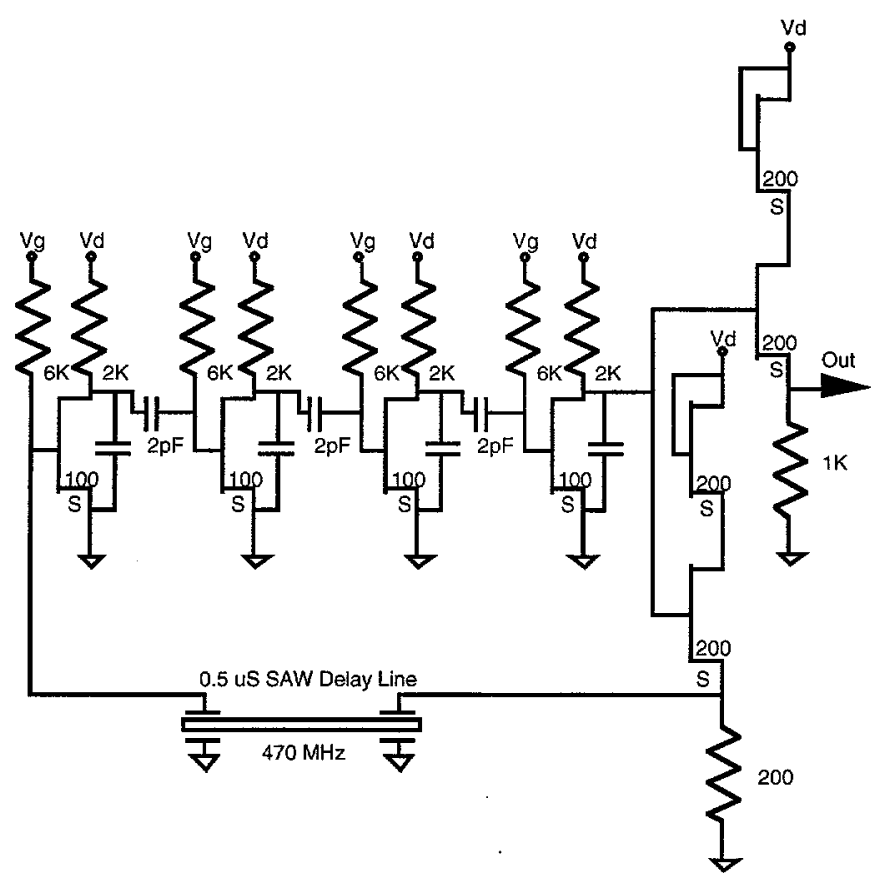

Figure 4. 


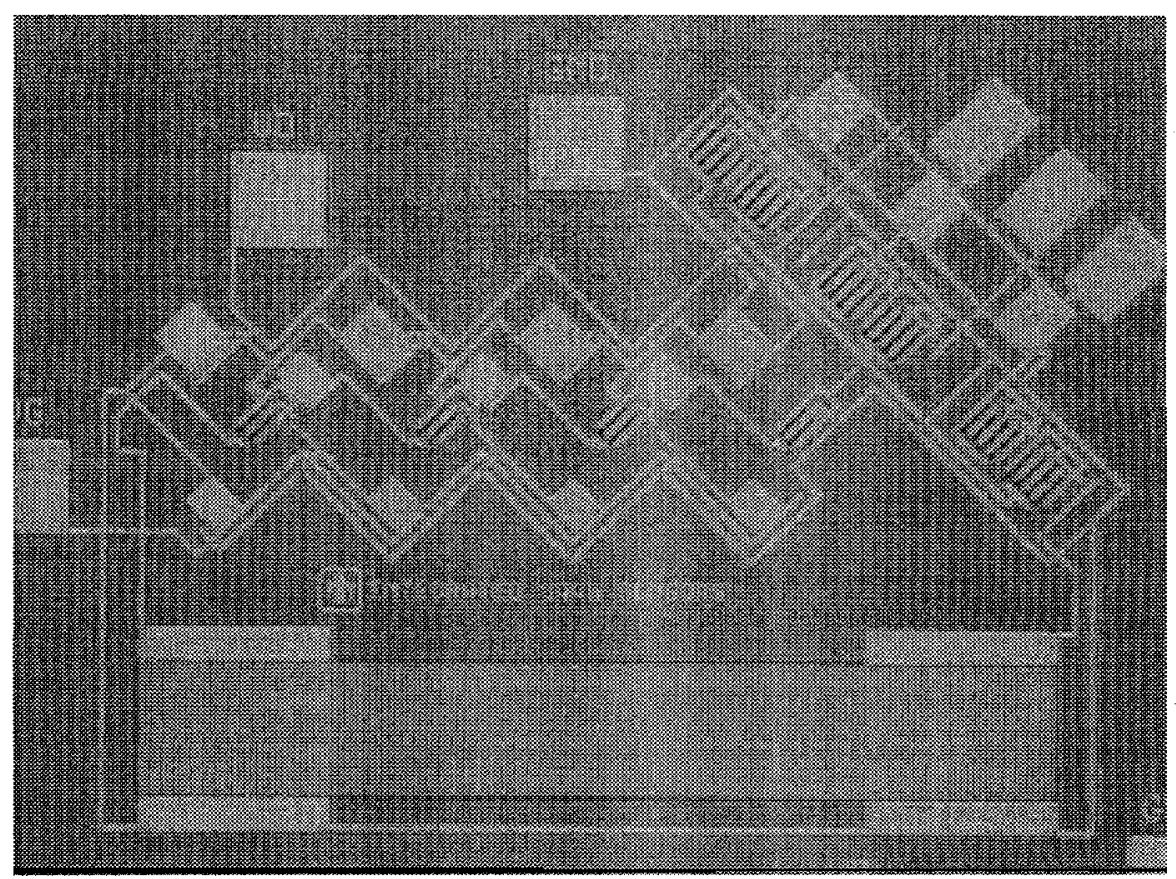

Figure 5. 


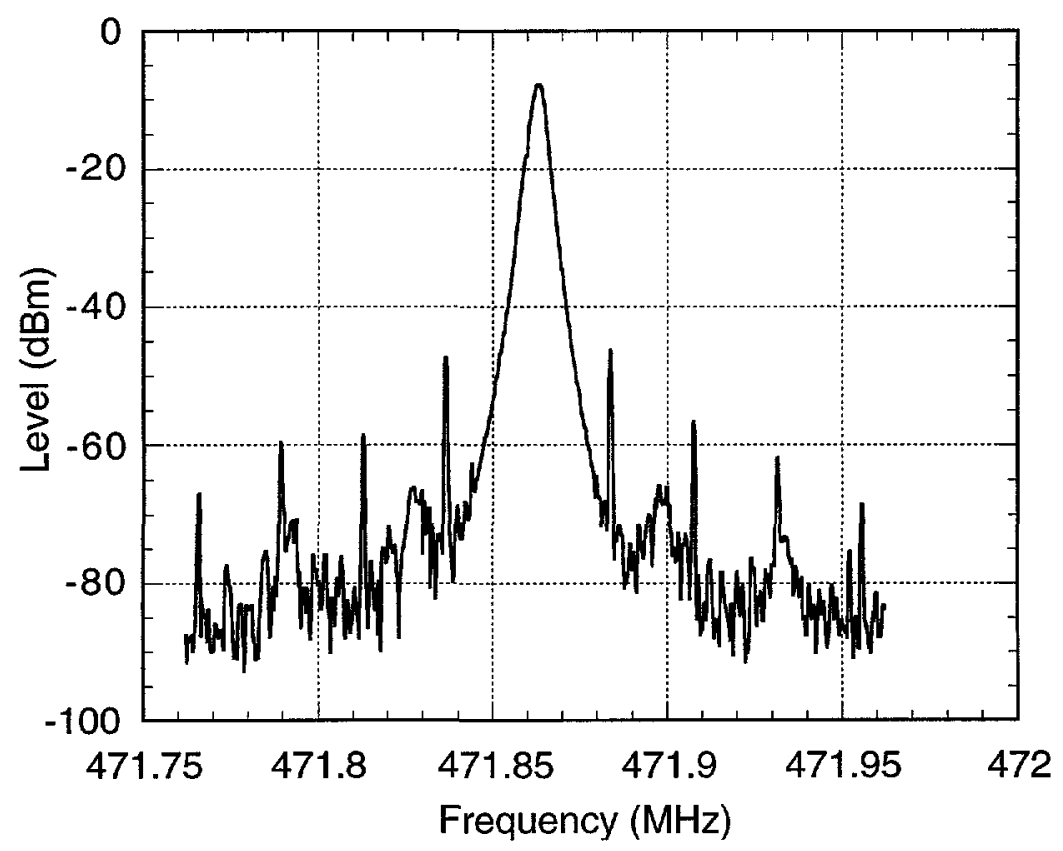

Figure 6. 